Theiler-Scherrer, Käthi; Senn Keller, Corinne

\title{
Simulationsspiel als subjektorientierte Konsumbildung
}

Haushalt in Bildung \& Forschung 3 (2014) 3, S. 75-90

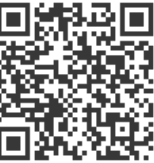

Quellenangabe/ Reference:

Theiler-Scherrer, Käthi; Senn Keller, Corinne: Simulationsspiel als subjektorientierte Konsumbildung In: Haushalt in Bildung \& Forschung 3 (2014) 3, S. 75-90 - URN: urn:nbn:de:0111-pedocs-204271 - DOI: 10.25656/01:20427

https://nbn-resolving.org/urn:nbn:de:0111-pedocs-204271

https://doi.org/10.25656/01:20427

in Kooperation mit / in cooperation with:

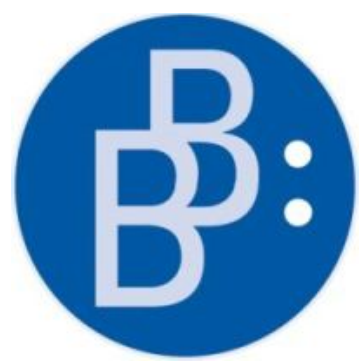

https://www.budrich.de

\section{Nutzungsbedingungen}

Gewährt wird ein nicht exklusives, nicht übertragbares, persönliches und beschränktes Recht auf Nutzung dieses Dokuments. Dieses Dokument ist ausschließlich für den persönlichen, nicht-kommerziellen Gebrauch bestimmt. Die Nutzung stellt keine Übertragung des Eigentumsrechts an diesem Dokument dar und gilt vorbehaltlich der folgenden Einschränkungen: Auf sämtlichen Kopien dieses Dokuments müssen alle Urheberrechtshinweise und sonstigen Hinweise auf gesetzlichen Schutz beibehalten werden. Sie dürfen dieses Dokument nicht in irgendeiner Weise abändern, noch dürfen Sie dieses Dokument für öffentliche oder kommerzielle Zwecke vervielfältigen, öffentlich ausstellen, aufführen, vertreiben oder anderweitig nutzen.

Mit der Verwendung dieses Dokuments erkennen Sie die Nutzungsbedingungen an.

\section{Terms of use}

We grant a non-exclusive, non-transferable, individual and limited right to using this document.

This document is solely intended for your personal, non-commercial use. Use of this document does not include any transfer of property rights and it is conditional to the following limitations: All of the copies of this documents must retain all copyright information and other information regarding legal protection. You are not allowed to alter this document in any way, to copy it for public or commercial purposes, to exhibit the document in public, to perform, distribute or otherwise use the document in public.

By using this particular document, you accept the above-stated conditions of use.

\section{Kontakt / Contact:}

\section{peDOCS}

DIPF | Leibniz-Institut für Bildungsforschung und Bildungsinformation Informationszentrum (IZ) Bildung

E-Mail:pedocs@dipf.de

Internet: www.pedocs.de

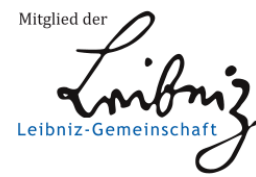


3. Jahrgang Heft 3

2014

는

๓)

$\bullet$

(1)

$\frac{1}{\bar{E}}$

0

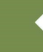

(1)

(

+

ᄃ

믕

은

논

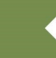

๕

(1)

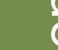

$\varepsilon$

ह

$\frac{\mathcal{B}}{\mathrm{N}}$

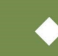

$+$

$\stackrel{\$}{ٍ}$

$\bullet$

$\frac{1}{\Phi}$
$\frac{c}{0}$
$\frac{5}{5}$
d
0

\section{Haushalt in Forschung}

\section{Subjektorientierung in der}

\section{Ernährungs- und Verbraucherbildung}


Inhaltsverzeichnis |

Silke Bartsch \& Claudia Wespi

Editorial. 2

Silke Bartsch \& Barbara Methfessel

Der „subjektive Faktor“. Bildung in einem lebensweltorientierten Fach 3

Werner Brandl

Bausteine und Baustelle einer Didaktik subjektorientierten Lernens und Lehrens... 33

Claudia Wespi \& Corinne Senn Keller

Subjektorientiertes Lernen und Lehren in einer kompetenzorientierten

Unterrichtskonzeption

Käthi Theiler-Scherrer \& Corinne Senn Keller

Simulationsspiel als subjektorientierte Konsumbildung 75

Barbara Methfessel \& Bärbel Schön

Biographisch orientierte Lehr-Lernprozesse als „Brücke“ zwischen

lebensweltlichen Erfahrungen und systematischen Lehr-Lernprozessen -

Erfahrungen aus einem Lehr-Forschungsprojekt.

Nicola Kluß

Ernährungswissen und -handeln am Beispiel von Vollkorn - ein Lehr-

Forschungsprojekt.

Werner Brandl

Rezension: Didaktische Prinzipien.

Melanie Lukas

Rezension: Wesen und Wege nachhaltigen Konsums. 
Subjektorientierte Konsumbildung |

Käthi Theiler-Scherrer \& Corinne Senn Keller

\section{Simulationsspiel als subjektorientierte Konsumbildung}

Im Planspiel Shoppingcenter werden Kaufentscheidungen simuliert, indem Aufgaben gelöst und reflektiert werden. Am Ende erfolgt eine Reflexion, welche v. a. den Lernprozess, resp. den Lernzuwachs des Subjekts fokussiert. Anlage und Anleitung eines Simulationsspiels scheinen auf den ersten Blick komplex, versprechen lebensnahe Lernauseinandersetzungen, die Anforderungen einer Subjekt- und einer Kompetenzorientierung einlösen können.

Schlüsselwörter: Lebensweltbezug, Handlungsorientierung, Perspektivenwechsel, Planspiel, Einkaufswelten

\section{Einführung}

Die grossen Einkaufszentren in der Schweiz heissen Tivoli, Shoppyland, Westside oder Sihlcity. Sie stehen für eine urbane und moderne Konsumkultur und können den Vergleich mit den amerikanischen Vorbildern, den Shopping Malls, aufnehmen. Für das vorliegende Planspiel dienen die Shoppingcenter mit vielversprechenden Namen als Spielszenario und bilden den imaginären Raum in dem Aufgaben gestellt und Kaufentscheidungen getroffen werden.

Dieser Beitrag zeigt eine Möglichkeit auf, wie Konsumbildung konkret umgesetzt werden kann. Das Spiel wurde so konzipiert, dass es in der Lehrerinnen- und Lehrerausbildung und mit Anpassungen in der Weiterbildung von Lehrpersonen eingesetzt werden kann. Dort soll es für Schülerinnen und Schüler der Sekundarstufe I von den Lehrpersonen selber weiter bearbeitet werden.

Zudem werden Bezüge zu den neusten Lehrplanentwicklungen in der Schweiz hergestellt. Der Deutschschweizer Lehrplan 21 (Deutschschweizer Erziehungsdirektorenkonferenz, 2014), der bis Ende 2014 fertiggestellt sein soll, zielt auf einen kompetenzorientierten Unterricht ab. Das Unterrichtskonzept von Landwehr (2008) möchte aufzeigen, wie ein kompetenzorientierter Unterricht aufgebaut werden kann und fokussiert die Subjektorientierung in einer konkreten Unterrichtsgestaltung.

Inwieweit ein Simulationsspiel diese Anforderungen erfüllen kann und welche Zusammenhänge zur Subjektorientierung als didaktischem Prinzip bestehen, werden ebenfalls beschrieben. Der Beitrag möchte zudem auf Herausforderungen und Gelingensbedingungen hinweisen. 


\section{Simulationsspiel in der Ausbildung von Lehrerinnen und Lehrer}

Das Planspiel Shoppingcenter wird im Folgenden zusammenfassend beschrieben und dessen Einsatz in der Ausbildung verortet. Die Spielunterlagen und der Spielverlauf werden mit ausgewählten Aspekten zur Theorie von Planspielen beleuchtet, insbesondere unter Berücksichtigung der Subjekt-, Kompetenz- und Erkenntnisorientierung.

\subsection{Fachwissenschaftliche Veranstaltung zur Konsumbildung}

Im Rahmen des Studiums zur Lehrperson Sekundarstufe 1 besuchen die Studierenden an der PH FHNW fachdidaktische und fachwissenschaftliche Veranstaltungen. Die Ausbildung ist modular aufgebaut. Dies bedeutet, dass die Studierenden nicht zwingend die empfohlene Reihenfolge der Module besuchen, sondern, dass sie auch vertiefende Angebote vor den Grundlagenmodulen in ihrem individuellen Studienplan belegen. Studierende innerhalb einer Veranstaltung bringen also häufig unterschiedliche fachliche Kompetenzen mit. Die folgenden Ausführungen beziehen sich auf eine fachwissenschaftliche Veranstaltung unter dem Titel „Haushalte als Konsumenten“, die für die Vertiefungsphase vorgesehen ist. Der Wissensaufbau erfolgt zu Themen wie Wirtschaftskreislauf aus der Perspektive der Haushalte, Haushaltstheorie, Konsumverhalten und -entscheidungen, Konsumentenverantwortung und Einflüsse des Marketings. Je nach individuellem Studienplan bringen die Studierenden Kompetenzen im Bereich Nachhaltigkeit mit. Nach dem Wissensaufbau zum Schwerpunkt Konsum folgt eine Sequenz mit dem Planspiel Shoppingcenter. Die Studierenden sollen das Fachwissen an konkreten Aufgaben anwenden und reflektieren und so ihr Können unter Beweis stellen. Die Methode Planspiel wird in Absprache mit der Fachdidaktik eingesetzt. So soll nebst dem fachlichen Kompetenzaufbau das Planspiel als Methode erlebt und für fachdidaktische Fragestellungen genutzt werden (pädagogischer Doppeldecker).

\subsection{Spielverlauf im Planspiel Shoppingcenter}

Die Hälfte der Jugendlichen in der Schweiz gibt laut James-Studie 2012 (Willemse et al., 2012) „Shoppen“ als regelmässige Freizeitbeschäftigung an. Wobei dies mehr Mädchen als Jungen tun. Zudem treffen sich drei Viertel aller Jugendlichen gerne mit Freunden. In Einkaufszentren kann beobachtet werden, wie Jugendliche sich in Gruppen insbesondere bei Bekleidungs- und Elektronikgeräteanbietern aufhalten. Genauso sind sie bei Verpflegungsangeboten und Aufenthaltsflächen wie Sitzgruppen, Treppen und Galerien anzutreffen. Das Planspiel Shoppingcenter greift dies auf und führt die Spielenden in der Rolle verschiedener Jugendlicher im Alter von 16-19 
Jahren durch ein virtuelles Einkaufszentrum zu bekannten, aber auch weniger beachteten Orten der Konsumwelt.

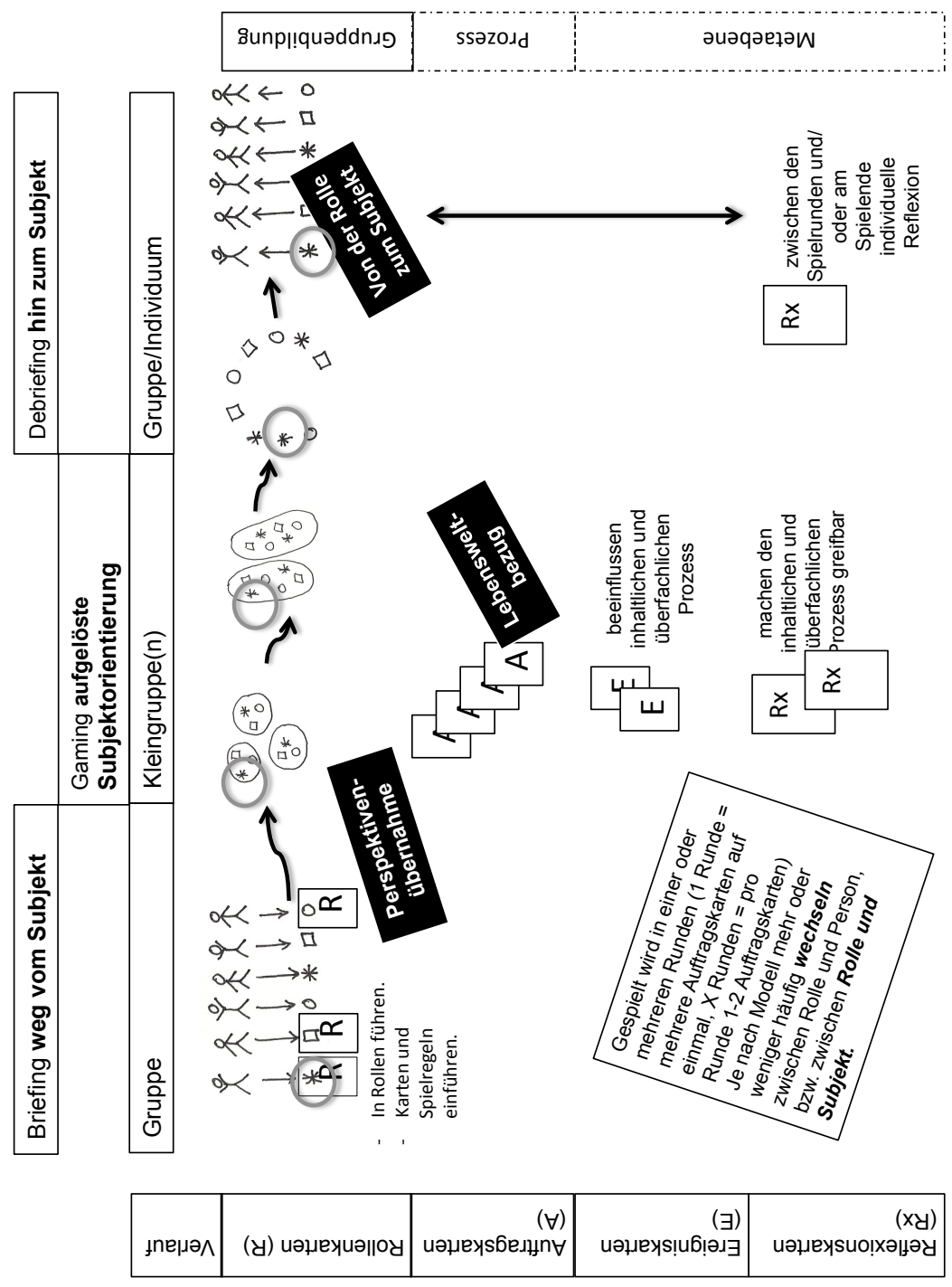

Abb. 1: Spielanlage und Spielverlauf im Planspiel „Shoppingcenter“ - hervorgehobene Elemente weisen auf Aspekte der Subjektorientierung hin (Senn Keller \& TheilerScherrer, 2013). 


\section{Subjektorientierte Konsumbildung}

In Abbildung 1 sind die Spielkarten, der Spielverlauf und bedeutsame Ebenen visualisiert. Zur besseren Orientierung ist ein Spieler durchgehend hervorgehoben.

\begin{tabular}{|c|c|}
\hline $\begin{array}{l}\text { Sozialökologisch } \\
\text { - distanziert sich von mat } \\
\text { - Verzicht in der Überfluss } \\
\text { - Werte wie Demokratie, } \\
\text { - versuchen andere für ih } \\
\text { - interessieren sich für Po }\end{array}$ & $\begin{array}{l}\text { ialistischen Werten } \\
\text { yesellschaft ein Gebot } \\
\text { erechtigkeit und Nachhaltigkeit sind bedeutsam } \\
\text { Ansichten zu gewinnen } \\
\text { tik und Gesellschaft }\end{array}$ \\
\hline Name & Barbara Bleuer \\
\hline Geschlecht, Alter & weiblich, 18 Jahre \\
\hline Schule/Beruf/Tätigkeit & $\begin{array}{l}\text { Gymnasiastin } \\
\text { möchte Umweltwissenschaft oder Politologie studieren }\end{array}$ \\
\hline $\begin{array}{l}\text { Herkunftsfamilie } \\
\text { - Berufe der Eltern } \\
\text { - Wohnort } \\
\text { - Geschwister } \\
\text { - Interessen }\end{array}$ & $\begin{array}{l}\text { - Vater, Projektleiter in Industriebetrieb } \\
\text { - Mutter arbeitet Teilzeit als Lehrerin } \\
\text { - Leben im Stadtbezirk West mit guter Anbindung an ÖV und viel } \\
\text { - Grünfläche } \\
\text { - Schwester, } 20 \text { in Ausbildung } \\
\text { - engagiert sich in der IG ,Gutes Leben im Stadtbezirk West' }\end{array}$ \\
\hline $\begin{array}{l}\text { Freundeskreis } \\
\text { Freizeitverhalten }\end{array}$ & $\begin{array}{l}\text { - trifft sich gerne mit Freunden, die sich auch für sozialökologische und } \\
\text { kulturelle Themen interessieren. } \\
\text { - geniesst Momente der Ruhe. } \\
\text { - geht gerne ins Theater und besucht interessante Ausstellungen in } \\
\text { Museen. } \\
\text { - belegt eine zweite Fremdsprache aus dem Freifachangebot. } \\
\text { - nutzt Notebook und Handy für ihre Anliegen (Informationssuche und } \\
\text { Informationsaustausch). }\end{array}$ \\
\hline $\begin{array}{l}\text { Bevorzugte Konsumgü- } \\
\text { ter }\end{array}$ & $\begin{array}{l}\text { Theater, Musik, Ausstellungen; No Name Kleidung; Qualität vor } \\
\text { Quantität }\end{array}$ \\
\hline Finanzielle Situation & \\
\hline Einnahmen monatlich & \\
\hline - Taschengeld & Fr. 180.- (inkl. Kleidergeld) \\
\hline - Nebenjobs & Fr. 200.- (arbeitet im Bio-Supermarkt) \\
\hline - Lehrlingslohn & \\
\hline Ausgaben monatlich & \\
\hline - Abgaben Wohnort & \\
\hline - Sparen & \\
\hline - Abzahlung/Schulden & \\
\hline - & \\
\hline
\end{tabular}

Abb. 2: Beispiel einer Rollenkarte (Senn Keller \& Theiler-Scherrer, 2013)

In der ersten Spielphase, dem Briefing (vgl. Capaul \& Ulrich, 2010), wird den Spielenden das virtuelle Shoppingcenter vorgestellt und eine Rolle zugeteilt, Spielgruppen (Kleingruppen) werden gebildet und allgemeine Regeln durch die Spielleitung bekannt gegeben. Die Rollen orientieren sich an den Typen der Sinusmilieus ${ }^{1}$. Damit sich die Spielenden in ihre Rolle versetzen können, werden sie aufgefordert 


\section{Subjektorientierte Konsumbildung}

anhand der Informationen auf der Rollenkarte $(\mathrm{R})$ einen für die Rolle typischen Tagesablauf zu beschreiben (vgl. Abb. 2). Mit diesem stellen sie sich dann auch ihrer Kleingruppe vor.

In der eigentlichen Spielphase, dem Gaming (vgl. Capaul \& Ulrich, 2010), treffen die Gruppen Konsumentscheidungen ausgehend von Auftragskarten (A), mit mehr oder weniger offenen Aufträgen. Berücksichtigt werden die Konsumfelder Finanzen und Dienstleistungen, Kleidung und Mode, Wohnen und Geräte, Esskultur und Ernährung sowie Freizeit und Sport. So suchen die Spielenden beispielsweise ein passendes Ferienangebot für eine gemeinsame Reise im Sommer und begründen die Wahl (Abb. 3). Oder sie beraten sich gegenseitig zu Bankkonten und wählen ein Angebot mit bedarfsgerechten Dienstleistungen aus. Für die Reklamation zu einem schlecht gereinigten Schlafsack informieren sie sich über ihre Rechte und formulieren ihre Forderungen in einem Reklamationsschreiben.

\begin{tabular}{|c|c|}
\hline & Freizeit und Sport \\
\hline Titel & Bedürfnisorientiert reisen \\
\hline Kompetenz & $\begin{array}{l}\text { Sie können eine Ferienreise planen unter Berücksichtigung des vorhandenen } \\
\text { Budgets, der Bedürfnisse der Gruppe und Kriterien der Nachhaltigkeit. }\end{array}$ \\
\hline Ausgangslage & $\begin{array}{l}\text { Sie planen mit ihrer Gruppe einen Badeurlaub. Über das Reiseziel sind Sie } \\
\text { sich noch nicht einig. Spanien, Türkei und Italien stehen zur Auswahl. Damit } \\
\text { Sie die Ferienansprüche am Arbeitsplatz rechtzeitig einreichen können, setzen } \\
\text { Sie sich jetzt zusammen. }\end{array}$ \\
\hline Auftrag & $\begin{array}{l}\text { Sie besprechen im Voraus: } \\
\text { - das Budget } \\
\text { - Bedürfnisse und Wünsche } \\
\text { - einen Aspekt der Nachhaltigkeit, den Sie berücksichtigen wollen } \\
\text { Jeder Mitspieler/jede Mitspielerin wählt zwischen Reisebüro und/oder Internet } \\
\text { und stellt einen Vorschlag zusammen. }\end{array}$ \\
\hline Produkt/Ergebnis & $\begin{array}{l}\text { Sie stellen den Kollegen/Kolleginnen gegenseitig die Reisevorschläge vor. } \\
\text { Gemeinsam entscheiden Sie sich für ein Angebot und begründen die Wahl. }\end{array}$ \\
\hline Material & 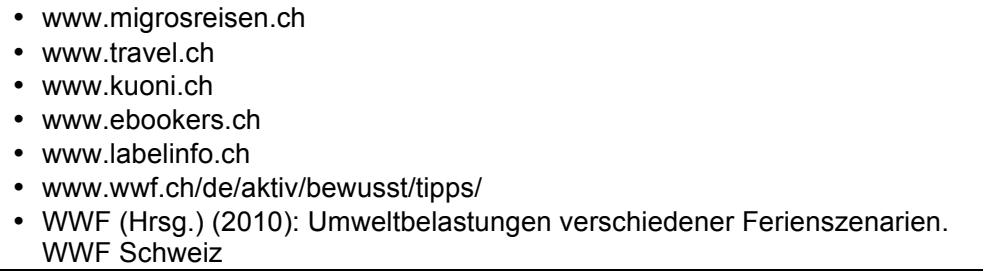 \\
\hline
\end{tabular}

Abb. 3: Beispiel einer Auftragskarte (Senn Keller \& Theiler-Scherrer, 2013)

Die Auftragskarten beinhalten Hinweise zu geeigneten Quellen. Die Spielenden sind aber auch aufgefordert, nach Interesse und Spielverlauf weitere Informationen zu beschaffen. Durch die unterschiedlichen Rollen innerhalb der Gruppen werden Entscheidungskonflikte ausgelöst. Verschiedene Argumente aus der Perspektive verschiedener Milieu-Typen kommen zur Sprache. Unterschiedliche Bedürfnisse 


\section{Subjektorientierte Konsumbildung}

und Interessen treffen aufeinander. Die Auftragskarte ist dann erfüllt, wenn die begründete(n) Entscheidung(en) und wesentliches Fachwissen festgehalten und an der Steckwand für die anderen Gruppen sichtbar publiziert sind. Nebst schriftlichen Formen gibt es auch Aufträge, bei denen beispielsweise eine Tonaufnahme eines Verkaufsgesprächs gefordert ist. In diesem Fall wird der Hinweis auf die Tonaufnahme publiziert.

Die Spielleitung bestimmt, ob und welche Reihenfolge bei den Spielkarten zu befolgen ist, wie viel Zeit für eine Karte zur Verfügung steht und wie viele Karten mindestens gespielt werden müssen. Bei Bedarf setzt sie Ereignis- und Reflexionskarten ein. So kann sie zum Beispiel bei der Wahl nach dem passenden Ferienangebot den Aspekt „sicher Einkaufen im Internet“ mit einer Ereigniskarte einbringen, falls dies die Spielenden ausser Acht lassen. Wenn der Prozess bei der Suche nach dem geeigneten Bankkonto stockt, setzt die Spielleitung eine Reflexionskarte ein und fordert damit die Spielenden auf, kurz innezuhalten und sich über ihr weiteres Vorgehen abzusprechen.

Am Ende einer Gamingphase vergleichen jeweils zwei Gruppen, die mit derselben Spielkartenauswahl gearbeitet haben, ihre Konsumentscheidungen. Von Interesse ist dabei vor allem, inwiefern sich die Konsumentscheidungen unterscheiden und mit welchen Perspektiven und Argumente diese zustande gekommen sind. So werden also beispielsweise die Ferienreisen oder die Reklamationsschreiben verglichen. Das Debriefing bildet den Abschluss. Zuerst in der ganzen Gruppe der Studierenden, dann aber auch individuell wird der fachliche und überfachliche Prozess reflektiert. Anhand eines Fragebogens erfassen die Studierenden ihre Erfahrungen zu Fachinhalten wie auch zu überfachlichen Aspekten und zum Spielverlauf. Insbesondere werden sie hier auch aufgefordert, die Konsumsituationen und -entscheidungen aus ihrer realen Perspektive zu analysieren. Wie würden sie selbst entscheiden?

Das Spiel wird idealerweise in mehreren Runden mit jeweils einer oder mehreren Spielkarten gespielt. So kann die Spielleitung bei Bedarf den Prozess insbesondere auf der fachlichen Ebene steuern und gezielt auf den Bedarf an Fachwissen eingehen zu Themen, die die Studierenden durch ihre Auseinandersetzung aufwerfen oder die aus der Sicht der Dozentin resp. des Dozenten wesentlich sind.

Das Planspiel berücksichtigt folgende Bildungsziele nach REVIS: „Die Schülerinnen und Schüler ...

- treffen Konsumentscheidungen reflektiert und selbstbestimmt.

- gestalten die Konsumentenrolle reflektiert in rechtlichen Zusammenhängen.

- treffen Konsumentscheidungen qualitätsorientiert.

- entwickeln einen nachhaltigen Lebensstil“ (REVIS-Fachgruppe, 2005). 


\section{Subjektorientierte Konsumbildung}

Aus dem LP 21 (Deutschschweizer Erziehungsdirektorenkonferenz, 2013a) berücksichtigt das Spiel aus den Kompetenzbereichen „Konsum und Lebensstil gestalten“ und „Märkte und Handel verstehen - über Geld nachdenken“ die folgenden Kompetenzen:

Die Schülerinnen und Schüler können ...

- Einflüsse auf Konsumgewohnheiten und Konsumhandlungen reflektieren.

- Folgen des Konsums analysieren.

- Konsumentscheide abwägen, reflektieren und verantwortlich handeln.

- das Zusammenspiel von Angebot und Nachfrage von Gütern und Dienstleistungen auf Märkten erklären.

- einen verantwortungsvollen Umgang mit Geld entwickeln.

\subsection{Theoretischer Hintergrund von Simulationsspielen}

Die Spielenden „erfahren im Plan-/Simulationsspiel einen ausgewählten Teil der Wirklichkeit sehr direkt, indem sie sich aktiv an der Simulation der Wirklichkeit beteiligen" (Capaul \& Ulrich, 2010, S. 17). Planspiele fordern auf, Entscheidungen zu treffen und soweit möglich Konsequenzen im simulierten System zu erfahren. Sie können laut Hense \& Mandl (2012) als problemorientierte Lernumgebungen betrachtet werden. Hervorzuheben sind die authentischen und anwendungsnahen Lernanlässe, sowie der Einbezug von multiplen Perspektiven und die Einbettung des Lernens in soziale Kontexte (vgl. Hense \& Mandl, 2012). Das Simulationsspiel geht davon aus, dass die Lernenden aktive Mitgestalter ihres Lernprozesses sind und die geforderten Aufgaben möglichst selbstständig lösen können. Landwehr (2008) geht davon aus, dass ihr subjektives Wissen mit neuem Wissen verknüpft wird und in einer Problemsituation zur Anwendung kommen kann.

Ausgangspunkt des vorgestellten Planspiels ist die komplexe Wirklichkeit der Konsumwelt. Im Zentrum der Simulation steht der Lebensweltbezug Jugendlicher, die ihre Freizeit im Shoppingcenter verbringen und Konsumentscheidungen treffen. Mit Rollen, Regeln und Aufträgen werden reale Vorgänge in reduzierter Komplexität und ohne reale Gefahren - wie beispielsweise zu viel Geld auszugeben simuliert. Für den Grad der Abstraktion ist „die didaktisch angemessene Reduktion der Realität" (Kriz, 2011, S. 16) entscheidend. So werden im vorliegenden Spiel jeweils nur eine Auswahl der Sinusmilieu-Typen ${ }^{2}$ pro Spielgruppe berücksichtigt, so dass die Spielenden zwar mit unterschiedlichen Perspektiven konfrontiert werden, sie aber gleichzeitig gemeinsam Konsumentscheidungen aushandeln können. Dafür wird auch mit Rollenkarten mit Jugendlichen über 18 Jahren gespielt, damit der Einsatz von Kreditkarten und die Möglichkeit Verträge abzuschliessen einbezogen werden kann. Preise und Budget werden bei verschiedenen Auftragskarten thematisiert, aber es ist kein Geld im Spiel. 


\section{Subjektorientierte Konsumbildung}

Der Spielverlauf erfolgt wie in Abbildung 1 dargestellt in den drei Phasen Briefing, Gaming und Debriefing (vgl. auch Capaul \& Ulrich, 2011, S. 18). Erst durch das Zusammenspiel dieser Phasen wird Lernen ermöglicht, wenn zwischen „den Ebenen der unmittelbaren Erfahrung auf die Ebene der Reflexion und des Lernens“ gewechselt wird (Hense \& Mandl, 2012, S. 16). Um Lernen zu ermöglichen, muss also nach dem Gaming zur Metaebene und der Reflexion im Debriefing gewechselt werden und müssen idealerweise die Erkenntnisse daraus im Briefing und Gaming der nächsten Spielrunde wieder einfliessen.

In der ersten Phase - dem Briefing - werden die Spielenden eingeführt in die Rahmensituation, informiert über die Spielregeln und den organisatorischen Rahmen und insbesondere zur Übernahme der Rolle angeleitet. Dies ermöglicht, Distanz zum Subjekt zu bekommen, andere als nur die eigene Perspektive einzunehmen und von sich unabhängigere Entscheidungen $\mathrm{zu}$ treffen. So entscheidet sich die Spielende in der Rolle der „Sozialökologischen Spielerin“ (vgl. Abb. 2) vielleicht für nachhaltige Ferien, auch wenn sie dies in der Realität nicht tun würde. Die Spielerin diskutiert mit Mitspielenden in der Rolle verschiedener Sinusmilieus, mit denen sie im Alltag weniger Kontakt hat. Dabei kann sie mit Argumenten konfrontiert werden, die sie bisher gar nicht beachtet hat und die auch im Widerspruch zu den eigenen stehen können. Innerhalb des Spiels und in der Rolle können die unterschiedlichen Argumente eher zugelassen und auch diskutiert werden.

Im Gaming stehen Aufträge aus der Lebenswelt der Jugendlichen im Zentrum. Der konstruierte Lebensweltbezug ist nicht bei allen Karten gleichermassen authentisch (vgl. Kiper et al., 2010). Es geht darum, den Spielenden auch Lernaufgaben ,abseits von Standard- und Routineaufgaben“ anzubieten und ihnen damit Lernsituationen zu ermöglichen ,die versuchen, die komplexe Wirklichkeit widerzuspiegeln“ (Tafner, 2012, S. 80), also beispielsweise das Einfordern der Konsumentenrechte bei einer Reklamation anhand der Textilreinigung durchzuspielen. Tafner (2012) sieht in diesem Sinne eine wesentliche Stärke des Planspiels im kompetenzorientierten Unterricht. Die Auftragskarten sind immer gleich strukturiert und beziehen Vorwissen und Präkonzepte (vgl. Bartsch \& Methfessel, i. d. H.) mit ein, indem sie beispielsweise das Wissen zu Nachhaltigkeit und die Vorgehensweise bereits gebuchter Reisen berücksichtigen.

In der dritten Phase - dem Debriefing - werden die Spielenden angeleitet, sich bewusst von ihrer Rolle und dem Spiel in der Gruppe zu verabschieden. Es geht darum, mit geeigneten Reflexions- und Moderationsmethoden „einmalige Spielerfahrungen zu generalisieren, das heisst in Beziehung zu typischen realen Verhaltensmustern zu setzen“ (Kriz, 2013, Position 2666). Im vorliegenden Spiel sollen die Studierenden einerseits die begründeten Konsumentscheidungen reflektieren und anderseits den gesamten Prozess im Planspiel analysieren. Ziel ist, ihre mentalen Modelle und subjektiven Handlungstheorien in Frage $\mathrm{zu}$ stellen (vgl. Kriz, 2013) und Erkenntnisse für das tatsächliche Konsumhandeln abzuleiten. Dazu müssen insbesondere die folgenden Fragen beantwortet werden: 


\section{Subjektorientierte Konsumbildung}

- Wie hast du dich gefühlt?

- Inwiefern unterscheidet sich das Planspiel von der Realität?

- Was habe ich dazugelernt?

- Wie würde ich als reale Person Konsumentscheidungen zu den Auftragskarten treffen?

- Welche Konsumentscheidungen treffe ich im Alltag? Wie habe ich die Entscheidungen bisher getroffen?

- Welche Erkenntnisse leite ich für zukünftige Konsumentscheidungen $\mathrm{ab}$ ?

\section{Subjektorientierung im Simulationsspiel}

Der folgende Abschnitt zeigt auf, mit welchem didaktischen Vorgehen subjektorientiertes Lernen und Lehren in der Planung von Unterricht integriert werden kann und wie das Simulationsspiel das aufgreift.

\subsection{Handlungs- und erkenntnisorientierte Didaktik}

Im erkenntnisorientierten Unterrichtskonzept von Landwehr (2008) werden drei Merkmale miteinander verbunden: die Prozessorientierung, die Problemorientierung und die Subjektorientierung.

Der Unterricht soll ermöglichen, dass Lernende den Lernstoff nicht nur aufnehmen und speichern. Vielmehr ist das Ziel, mit einer erkenntnisorientierten Wissensvermittlung neues Wissen in die kognitive Struktur des Lernenden zu integrieren. „Entsprechend ist im erkenntnisvermittelnden Unterricht nicht mehr die originalgetreue Reproduktion der Wissensinhalte das zentrale Erfolgskriterium für einen gelungenen Lehr- und Lernprozess, sondern die ,Evaluierung ' des bereits vorhandenen subjektiven Wissens der Lernenden und die daraus resultierende Umgestaltung und Weiterentwicklung des individuellen Denk- und Erfahrungshorizonts“ (Landwehr, 2008, S. 31).

Lernende sind dabei aktive Mitgestalter ihres eigenen Lernprozesses und Lehrpersonen werden dann eher zu Lernbegleiter. Der Bezugspunkt bildet dabei der subjektive Verarbeitungsprozess der Lernenden und das vorhandene subjektive Wissen ist zentraler Ausgangspunkt. Dabei wird das vorhandene subjektive Wissen verändert. Lernen in diesem Verständnis setzt voraus, dass die Lernenden das vorhandene subjektive Wissen aktivieren und mit dem vorliegenden Informationsangebot vergleichen und anschliessend das fehlende Wissen ergänzen oder korrigieren. Die Orientierung am Subjekt, wird dabei zur Notwendigkeit für einen erkenntnisorientierten Unterricht (Landwehr, 2008).

Die Planung des Unterrichts wird als problemorientierter Auseinandersetzungsprozess konzipiert. Damit der Lerninhalt verstanden werden kann, braucht es 


\section{Subjektorientierte Konsumbildung}

eine erkenntnisleitende Problemstellung, möglichst aus der Lebenswelt der Lernenden. Die Problemstellung, die am Anfang des Lernprozesses steht, soll bei den Lernenden einen kognitiven Konflikt auslösen. Lernen bedeutet für die Schülerinnen und Schüler, sich in einem ständigen Such- und Entdeckungsprozess zu befinden. Sie suchen selber aktiv nach geeigneten Lösungsmöglichkeiten und können die gewonnenen Erkenntnisse gegen Ende des Lernprozesses anwenden.

Das erkenntnisorientierte Unterrichtskonzept erfüllt wichtige Bedingungen subjektorientierten Lernens und Lehrens. Der Lebensweltbezug und die Aktivierung des Vorwissens der Schülerinnen und Schüler bilden den Beginn jeder Lernauseinandersetzung. So gesehen ist das Konzept des erkenntnisorientierten Unterrichts eine Möglichkeit, die Subjektorientierung konsequent im Unterricht zu integrieren (vgl. Wespi \& Senn, i. d. H.).

\subsection{Erkenntnisorientierung und Subjektorientierung im Planspiel}

Die Ausarbeitung eines Planspiels ist aufwändig und braucht grundsätzliche Vorüberlegungen. Der Zusammenhang zum problemorientierten, resp. erkenntnisorientierten Unterrichtskonzept wurde schon im Kapitel 2.3 vorgestellt. Ebenfalls wurde auf den Lebensweltbezug und die damit verbundenen authentischen und anwendungsnahen Lernanlässe hingewiesen. Im Weiteren werden die drei Phasen des Planspiels: Briefing, Gaming und Debriefing im Zusammenhang mit der Subjektorientierung untersucht.

Beim Briefing werden die Spielregeln vorgestellt und die Spielenden erhalten eine zugewiesene Rollenkarte (vgl. Abb 2.) Auf den ersten Blick widerspricht ein solches Vorgehen der Vorstellung, die Schülerinnen und Schüler in ihrem Lebensweltbezug und mit ihrem Vorwissen abzuholen. Eine Rolle einzunehmen kann jedoch Raum geben für Kreativität und Spontanität. Nicht alle Menschen können damit gleich gut umgehen. Dies gilt es zu berücksichtigen und braucht von Seiten der Spielleitung Begleitung. In eine Rolle schlüpfen zu können, kann aber auch schützend wirken, indem die eigene Privatheit nicht offen gezeigt werden muss. Bei der Konzeption des Planspiels wurde die Lebenswelt bei den Rollenkarten, insofern berücksichtigt, dass sie auf der Sinusmilieu Studie beruhen und auf die Zielgruppe der jungen Erwachsenen/Jugendlichen abgestimmt sind.

Während der Gaming-Phase müssen die Spielenden in ihrer Rolle Aufgaben lösen und Konsumentscheidungen treffen. Sie spielen in Kleingruppen und müssen sich gegenseitig absprechen. Dabei entsteht ein wechselseitiger Austausch, Interessenkonflikte müssen ausgetragen und Kompromisse ausgehandelt werden. Dadurch wird der von Landwehr (2008) beschriebene Such- und Entdeckungsprozess unterstützt (vgl. Kapitel 2.2.), indem neues Wissen beschafft und aufgebaut werden muss. Der Aushandlungsprozess hilft eigene Ansichten und Denkweisen sprachlich zu formulieren und seine Argumente stichhaltig und überzeugend in die Diskussion einzubringen. Die Phase des Gaming kann den kooperativen Lernformen zugeordnet 


\section{Subjektorientierte Konsumbildung}

werden und löst die Forderung ein, dass in einem kompetenzorientierten Unterricht fachliche und überfachliche Kompetenzen gelernt werden sollen. Die Ereigniskarten können den Spielverlauf unterstützen oder ihn unterbrechen. Die Spielleitung/Lehrperson hat die Befugnis in das Spielgeschehen einzugreifen und es zu beeinflussen. Im Kontext Schule und Unterricht soll der Einsatz dieser Karten immer „lernprozessdienlich“ gestaltet sein. Der Lebensweltbezug wird sichtbar, indem unvorhergesehene Ereignisse eingestreut werden, wie das im Leben auch geschehen kann. Am Ende der Gaming-Phase wird in der Kleingruppe über den fachlichen und überfachlichen Lernprozess reflektiert.

In der Debriefing-Phase wird das Spiel aufgelöst, indem über das Spielgeschehen in verschiedenen Formationen reflektiert wird. Dabei legen die Spielenden ihre Rolle ab. Mit Hilfe eines Fragebogens überlegen sich die Spielenden, wie sie tatsächlich in ihrem Leben gehandelt hätten. Dabei knüpfen sie an ihre subjektiven Theorien an und überprüfen ihr vorhandenes Wissen und können es gegebenenfalls anpassen, Lernen wird sichtbar.

Ein erwähnenswerter Nebeneffekt des Planspiels bezieht sich auf die Anforderungen als angehende Lehrperson. Der in allen Fächern geforderte Lebensweltbezug ist nicht neu und wird auch nicht bestritten. Die Frage ist vielmehr, wie er im Unterricht und damit auch in konkreten Unterrichtsplanungskonzepten eingelöst werden kann. Diesbezüglich schlagen Kattmann et al. (1997) mit der didaktischen Rekonstruktion einen gangbaren Weg vor (vgl. Wespi \& Senn, i. d. H.).

Wenn Studierende eine Rolle einnehmen müssen, die ihrer Lebenswelt und ihrem Milieu gar nicht entspricht, können die Erlebnisse im Spiel und der Perspektivenwechsel, den Zugang zur Lebenswelt der Schülerinnen und Schüler unterstützen. Die Studierenden können Verständnis und Sensibilität entwickeln und diese dann im besten Fall bei ihrer zukünftigen Unterrichtsplanung berücksichtigen.

\section{Simulationsspiele im Unterricht}

In diesem Abschnitt wird ein Bezug zu den neusten bildungspolitischen Entwicklungen in der Schweiz hergestellt. Mit der Implementierung eines kompetenzorientierten Lehrplans werden von den Lehrpersonen veränderte Unterrichtskonzepte erwartet. Das Simulationsspiel Shoppingcenter kann dazu ein Beitrag leisten.

\subsection{Kompetenzorientierung}

Die Forderung nach einer Konsumbildung, in der die Schülerinnen und Schüler befähigt werden, in unterschiedlichen Situationen und angesichts wechselnder Problemlagen als Konsumenten angemessen handeln zu können, wurde schon im Forschungsprojekt (REVIS-Fachgruppe, 2005) eingefordert. Die Verbraucherbildung wird aus der Perspektive der Menschen als handelnde Konsumenten betrachtet. Es geht dabei um die Interaktion zwischen Mensch und Markt. Das Konzept „Consumer 


\section{Subjektorientierte Konsumbildung}

Literacy“ zielt auf eine weitgehend verantwortungsvolle, gleichberechtigte, und erfolgreiche Teilhabe an der (Konsum)-Gesellschaft ab. Damit diese Teilhabe gelingt, braucht es im Umgang mit Konsumentscheidungen während der obligatorischen Schulzeit entsprechende Bildungsangebote (Schlegel-Matthies, 2004).

Der Lehrplan 21 basiert auf Kompetenzformulierungen über die gesamt Volksschulzeit (Vorschule bis zum 9. Schuljahr) und soll ab Ende 2014 alle bisherigen Lehrpläne in der Deutschschweiz ersetzen. Die Orientierung an Kompetenzen stellt andere Anforderungen an die Planung und Durchführung von Unterricht als die frühere Auflistung von Grobzielen und Inhalten in den Lehrplänen. Schülerinnen und Schüler sollen sich aktiv mit den Lerninhalten auseinandersetzen und dabei Wissen, Fähigkeiten und Fertigkeiten sowie Bereitschaften, Haltungen und Einstellungen entwickeln. Das bereits vorhandene Wissen und die gemachten Erfahrungen sind Ausgangspunkt für die Planung des Lernprozesses. Im Lehrplan sind für die verschiedenen Fachbereiche die Kompetenzerwartungen an Schülerinnen und Schüler definiert (Deutschschweizer Erziehungsdirektorenkonferenz, 2013b).

Schülerinnen und Schüler sind beispielweise in einem Fach kompetent, wenn sie

- zentrale fachliche Begriffe und Zusammenhänge verstehen, sprachlich zum Ausdruck bringen und in Aufgabenzusammenhängen nutzen können

- fachlich bedeutsame Fähigkeiten und Fertigkeiten zum Lösen von Problemen und zur Bewältigung von Aufgaben nutzen können

- auf vorhandenes Wissen zugreifen oder sich das notwendige Wissen beschaffen können

- ihr sachbezogenes Tun zielorientiert planen und in der Durchführung angemessene Handlungsentscheidungen treffen können

- Lerngelegenheiten aktiv und selbstmotiviert nutzen und dabei Lernstrategien einsetzen können

- fähig sind, ihre Kompetenzen auch in Zusammenarbeit mit anderen einzusetzen. (Deutschschweizer Erziehungsdirektorenkonferenz, 2013b)

Aufgaben im Unterricht sind wichtige Gestaltungselemente. Sie lassen zu, dass die Schülerinnen und Schüler individuelle Lernwege beschreiten können. In diesem Sinne erhält bei einem kompetenzorientierten Unterricht das Subjekt eine grössere Bedeutung. Das Lernen der einzelnen Schülerin, des einzelnen Schülers rückt bei der Planung und Durchführung von Unterricht viel stärker in den Vordergrund (Deutschschweizer Erziehungsdirektorenkonferenz, 2013b).

Das entwickelte Simulationsspiel erfüllt aus Sicht der Autorinnen wichtige Aspekte eines kompetenzorientierten Unterrichts. Vergleicht man die oben aufgeführten Ansprüche und allgemeinen Kompetenzziele mit der Spielanlage des Planspiels, werden weitgehend alle Punkte erfüllt. Einzig die Frage nach der Motivation und der 


\section{Subjektorientierte Konsumbildung}

Volition müsste noch genauer betrachtet werden, insbesondere auf der Ebene der Schülerinnen und Schüler.

\subsection{Rolle der Lehrperson}

Werden Planspiele im Unterricht oder in der Lehre eingesetzt, steht eigenverantwortliches Arbeiten und Lernen im Zentrum (vgl. Klippert, 2008). Dies bedeutet, dass die Lehrperson bzw. die Dozierende sich ihrer Rolle bewusst ist und darauf achtet, dass instruktionale Unterstützung sehr gezielt und insbesondere zurückhaltend eingesetzt wird (vgl. Hense \& Mandl, 2012). Im Vordergrund steht eine ressourcenorientierte Haltung der Spielleitung, die den Lernenden etwas zutraut und auch zumutet und sie wenn nötig berät. Fehler und Lernumwege werden zugelassen, die Lernenden werden durch Zielvorgaben und adaptive Lernbegleitung unterstützt (Klippert, 2008). Eine solche Haltung und Lernbegleitung ermöglicht Vorwissen und Präkonzepte der Lernenden gezielt einzubeziehen und der Subjektorientierung damit Rechnung zu tragen. Idealerweise verfügen die Lernenden bereits über Grundlagen überfachlicher Kompetenzen wie beispielsweise sich in Gruppen zu organisieren, selbstständig zu arbeiten und Verantwortung zu übernehmen. Dies sind hilfreiche Grundvoraussetzungen für das Simulationsspiel.

\section{Fazit und Ausblick}

Subjektorientiertes Lernen und Lehren wird im Unterrichtskonzept von Landwehr (2008) ausdifferenziert und ist somit für die Unterrichtsplanung und -durchführung handhabbarer. Simulationsspiele beinhalten Elemente einer Subjektorientierung, in dem sie einen ausgewählten Teil der Wirklichkeit darstellen und somit die Forderung nach einem Lebensweltbezug einlösen. Ebenso werden das Vorwissen und die Vorerfahrungen der Spielenden einbezogen. Während des Spiels werden anforderungsreiche Aufgaben bearbeitet. Dabei wird Fachwissen aufgebaut, das für die Problemlösung notwendig ist, aber auch darüber hinausgeht. Die Aufgaben fordern Entscheidungen heraus, die in Gruppen diskutiert und ausgehandelt werden. Das Gruppenresultat wird veröffentlicht, sodass andere Kleingruppen davon profitieren können. Ein solches Vorgehen entspricht einem erkenntnisorientierten Unterricht (Landwehr, 2008; vgl. Wespi \& Senn, i. d. H.) mit den Lernschritten: Problemkonfrontation, aktive Lösungssuche, Lösungsevaluation, Anwendung der Erkenntnisse.

In der Lehrerinnen- und Lehrerausbildung wird das Planspiel als Möglichkeit genutzt, das aufgebaute Wissen in konkreten Situationen zu üben und anzuwenden. Wie bei der Kompetenzorientierung gefordert, rückt Wissen und Können nahe zueinander oder wird sogar miteinander verbunden. Durch eine aktive Lernauseinandersetzung können die Studierenden ihren Lernstand erkennen, erweitern und reflektieren. Für Studierende entsteht somit ein pädagogischer Doppeldecker (Kriz, 2013). Durch die Selbsterfahrungen als Spieler oder Spielerin, können sie Fach- 


\section{Subjektorientierte Konsumbildung}

kompetenzen aufbauen und lernen zugleich eine interessante Methode kennen, die sie später in ihrem eigenen Unterricht anwenden können. Durch den Rollenwechsel wird ihnen bewusst, dass Menschen ihre Konsumentscheidungen auf Grund ihrer Herkunft unterschiedlich tätigen und begründen können. Dies eröffnet ihnen die Perspektive, dass ihre Schülerinnen und Schüler aus sehr unterschiedlichen Lebenswelten kommen und eine individuelle Lernauseinandersetzung und Lernbegleitung notwendig wird.

Die Erarbeitung eines Simulationsspiels erfordert einen hohen Zeitaufwand, sinnvollerweise sollte dies in Fachgruppen geschehen. In der Unterrichtspraxis kann sich die Lehrperson auf die Lernprozesse der Schülerinnen und Schüler konzentrieren und ihre Aufgabe als Lernbegleiterin resp. Lernbegleiter wahrnehmen. Das Simulationsspiel erfüllt somit wichtige Anforderungen eines kompetenzorientierten Unterrichts, indem die Lernenden eine hohe Eigenaktivität und individuelle Lernzeit haben. Sie werden herausgefordert selbstständig zu arbeiten, ihre eigenen Lernstrategien zu entwickeln und das neue Wissen indirekt/direkt anzuwenden. In den Aushandlungsprozessen werden überfachliche Kompetenzen wie Kommunikations-, Kooperations- und Konfliktfähigkeit gelernt.

„Subjektorientierung als didaktisches Prinzip trägt zu kompetenzorientiertem, salutogenetischem und lebensbegleitendem Lernen bei" (Bartsch, 2008, S. 100). Das Simulationsspiel Shoppingcenter ist ein methodisches Beispiel, wie man entsprechende, aktuelle Forderungen an einen modernen Unterricht einlösen kann.

\section{Anmerkungen}

1 „Sinusmilieus gruppieren Menschen, die sich in ihrer Lebensauffassung und Lebensweise ähneln“" (Thomas \& Calmbach, 2013, S. 18).

2 SINUS-Lebensweltenmodell U18 für 14-17-Jährige in D [www.sinus-akademie.de/fileadmin/user_files/Presse/SINUSJugendstudie_u18_2012/\%C3\%96ffentlicher_Foliensatz_Sinus-Jugendstudie_u18.pdf].

\section{Literatur}

aid (Hrsg.). (2013). REVIS - Ernährungs- und Verbraucherbildung im Unterricht. Bonn: aid Infodienst.

Bachner, J. \& Willnecker, M. (2011). „Der Weg ist das Ziel“ - Entwicklung eines Planspiels mit Nachwuchsführungskräften. In S. Hitzler, B. Zürn \& F. Trautwein (Hrsg.), Planspiel - Qualität und Innovation. Neue Ansätze aus Theorie und Praxis. (S. 145-168). ZMS-Schriftenreihe (Band 2).

Bartsch, S. (2008). Subjektorientierung in der Ernährungs- und Gesundheitsbildung. Ernährung - Wissenschaft und Praxis, 2 (3), 100-106. 


\section{Subjektorientierte Konsumbildung}

Birgmayer, R. (2011). Planspielleistung beurteilen - ein Widerspruch? In S. Hitzler, B. Zürn \& F. Trautwein (Hrsg.), Planspiel - Qualität und Innovation. Neue Ansätze aus Theorie und Praxis (S. 39-56). ZMS-Schriftenreihe (Band 2).

Capaul, R. \& Ulrich, M. (2010). Planspiele. Simulationsspiele für Unterricht und Training. Altstätten: Tobler Verlag.

Deutschschweizer Erziehungsdirektorenkonferenz (2013a). Lehrplan 21. Wirtschaft, Arbeit, Haushalt. Konsultationsversion vom Juni 2013.

[http://konsultation.lehrplan.ch/downloads/container/31_6_3_3_0_1.pdf].

Deutschschweizer Erziehungsdirektorenkonferenz (2013b). Lehrplan 21. Einleitung. Konsultationsversion vom Juni 2013.

[http://konsultation.lehrplan.ch/downloads/container/31_102_0_1_0.pdf]

Deutschschweizer Erziehungsdirektorenkonferenz (2014). Lehrplan 21.

[www.lehrplan.ch]

Fachgruppe Ernährungs- und Verbraucherbildung (2005). Schlussbericht REVIS Modellprojekt. Reform der Ernährungs- und Verbraucherbildung in Schulen. [www.ernaehrung-und-verbraucherbildung.de/evb_revis_schlussbericht.php].

Hense, J. \& Mandl, H. (2012). Curriculare Herausforderungen bei der Integration von Planspielen. In S. Schwägle, B. Zürn \& F. Trautwein (Hrsg.), Planspiel Lernen im Methoden-Mix. Integrative Lernkonzepte in der Diskussion (S. 1125). ZMS-Schriftenreihe (Band 4).

Kattmann, U., Duit, R., Gropengiesser, H. \& Komorek, M. (1997). Das Modell der Didaktischen Rekonstruktion - Ein Rahmen für naturwissenschaftsdidaktische Forschung und Entwicklung. Zeitschrift für Didaktik der Naturwissenschaften, 3 (3), 3-18.

Kiper, H., Meints, W., Peters, S., Schlump, S. \& Schmit, S. (2010). Lernaufgaben und Lernmaterialien im kompetenzorientierten Unterricht. Stuttgart: Kohlhammer.

Klippert, H. (2008). Planspiele. 10 Spielvorlagen zum sozialen, politischen und methodischen Lernen in Gruppen. Weinheim und Basel: Beltz Verlag.

Kriz, W. C. (2011). Qualitätskriterien von Planspielanwendungen. In S. Hitzler, B. Zürn \& F. Trautwein (Hrsg.), Planspiel - Qualität und Innovation. Neue Ansätze aus Theorie und Praxis (S. 11-37). ZMS-Schriftenreihe (Band 2).

Kriz, W. C. (2013). Erwerb von Systemkompetenz mit Planspielmethode. In H. Bachmann (Hrsg.), Hochschullehre variantenreich gestalten. Kompetenzorientierte Hochschullehre - Ansätze, Methoden und Beispiele. Forum Hochschuldidaktik und Erwachsenenbildung der Pädagogischen Hochschule Zürich. Bern: Hep-Verlag. E-book Position 2162-2887.

Landwehr, N. (2008). Neue Wege der Wissensvermittlung. Oberentfelden: Sauerländer.

Schlegel-Matthies, K. (2004). Verbraucherbildung im Forschungsprojekt REVIS Grundlagen. Paderborner Schriften zur Ernährungs- und Verbraucherbildung, 2/2004. Paderborn: Fachgruppe Ernährungs- und Verbraucherbildung. 


\section{Subjektorientierte Konsumbildung}

Senn Keller, C. \& Theiler-Scherrer, K. (2013). Planspiel Shoppingcenter. Basel und Brugg: Pädagogische Hochschule Nordwestschweiz FHNW.

Tafner, G. (2012). Integrative Wirtschaftsethik erleben. Das Planspiel im kompetenzorientierten Unterricht. In S. Schwägle, B. Zürn \& F. Trautwein (Hrsg.), Planspiel - Lernen im Methoden-Mix. Integrative Lernkonzepte in der Diskussion (S. 79-94). ZMS-Schriftenreihe (Band 4).

Thomas, P. M. \& Calmbach, M. (2013). Jugendliche Lebenswelten. Perspektiven für Politik, Pädagogik, Gesellschaft. Berlin Heidelberg: Springer Spektrum.

Willemse, I., Waller, G., Süss, D., Genner, S., Huber A.-L. (2012). JAMES - Jugend, Aktivitäten, Medien - Erhebung Schweiz. Zürich: Zürcher Hochschule für Angewandte Wissenschaften.

[www.jugendundmedien.ch/fileadmin/user_upload/Fachwissen/Ergebnisbericht_ JAMES_2012.pdf].

\section{Verfasserinnen}

Käthi Theiler-Scherrer

Corinne Senn Keller

Pädagogische Hochschule FHNW

Institut Sekundarstufe I und II

Professur Gesundheit und Hauswirtschaft

Clarastrasse 57

CH-4051 Basel

E-Mail: kaethi.theiler@fhnw.ch; corinne.sennkeller@fhnw.ch

Internet: www.fhnw.ch/ph und www.gesundheitundhauswirtschaft.ch 